\title{
Physical therapy management of Pompe disease
}

\author{
Laura Elizabeth Case, $P T, D P T^{1}$, and Priya Sunil Kishnani, $M D^{2}$
}

\begin{abstract}
Pompe disease (Glycogen storage disease type II, GSDII, or acid maltase deficiency) is an autosomal recessive disorder characterized by deficiency of acid $\alpha$-glucosidase resulting in intra-lysosomal accumulation of glycogen and leading to progressive muscle dysfunction. The natural history of infantile-onset Pompe disease is characterized by hypertrophic cardiomyopathy and profound generalized weakness presenting in the first few months of life, with rapid progression and death usually occurring by one year of age. Late-onset Pompe disease is characterized by onset of symptoms after one year of age, less severe or absence of cardiac involvement and slower progression, with symptoms primarily related to progressive dysfunction of skeletal muscles and respiratory muscle involvement. Recent clinical trials of enzyme replacement therapy have begun to allow greater opportunity for potential improvement in motor status, function, and survival than ever before, with hopes of moving toward maximizing physical function for individuals with Pompe disease. Children are living longer with some achieving independent sitting, creeping, and walking-milestones typically never achieved in the untreated natural history of the disorder. With increased survival, clinical management based on an understanding of the pathology and pathokinesiology of motor function gains importance. This article reviews current knowledge regarding the motor system in Pompe disease and provides an overview of physical therapy management of Pompe disease, including management strategies for individuals on enzyme replacement therapy.
\end{abstract}

Genet Med 2006:8(5):318-327.

Key Words: Pompe, glycogen storage disease, physical therapy, lysosomal storage disease, acid maltase deficiency

Pompe disease (Glycogen storage disease type II, GSDII, or acid maltase deficiency) is an autosomal recessive disorder characterized by deficiency of acid $\alpha$-glucosidase (GAA) resulting in intra-lysosomal accumulation of glycogen. ${ }^{1,2}$ Glycogen accumulates in various tissues, most significantly muscle, including cardiac, skeletal, and smooth muscle. ${ }^{1,2}$ The natural history of infantile-onset Pompe disease is characterized by hypertrophic cardiomyopathy and profound generalized weakness presenting in the first few months of life, with rapid disease progression and death usually occurring by one year of age. ${ }^{1,2}$ Late-onset Pompe disease (juvenile and adult onset) is characterized by onset of symptoms after one year of age, less severe to no cardiac involvement, and slower progression, with symptoms primarily related to progressive dysfunction of skeletal and respiratory muscles. ${ }^{1,3,4}$ The advent of enzyme replacement therapy (ERT) has begun to allow greater opportunity for potential improvement in motor status, function, and survival than ever before, with the hope of moving toward maximizing physical function for individuals with Pompe

\footnotetext{
From the ${ }^{1}$ Division of Physical Therapy, Department of Community and Family Medicine, School of Medicine, Duke University Medical Center, Durham, NC; ${ }^{2}$ Division of Medical Genetics, Department of Pediatrics, Duke University Medical Center, Durham, NC.

Laura E. Case, PT, DPT, MS, PCS, Clinical Associate in Division of Physical Therapy, Department of Community and Family Medicine, School of Medicine, Duke University Medical Center, Durham, NC,27707.E-mail: case0001@mc.duke.edu

Submitted for publication June 20, 2005.

Accepted for publication February 20, 2006

DOI: 10.1097/01.gim.0000217789.14470.c5
}

disease..$^{5-10}$ In this article the pathology and pathokinesiology of motor function in Pompe disease, and an overview of physical therapy (PT) management of Pompe disease, are provided. Studies have not previously been published on the use of PT with individuals with Pompe disease, therefore recommendations in this article are based on expert opinion, experience, and evidence from other neuromuscular disorders that have clinical features in common with Pompe disease.

\section{OVERVIEW OF MOTOR INVOLVEMENT}

\section{Pathology of muscle weakness}

Myopathy in Pompe disease results from intra-lysosomal accumulation of glycogen in muscle. ${ }^{1}$ Glycogen accumulates primarily in muscle (cardiac, skeletal, and smooth) and the liver, but also in the central nervous system (including anterior horn cells, motor nuclei of the brain stem, and spinal ganglia) and other tissues. ${ }^{1}$ Glucose metabolism is normal. ${ }^{1,2}$ Muscle involvement begins with enlargement of muscle fibers as glycogen accumulates in intact lysosomes, ${ }^{11}$ later followed by muscle wasting, the precise mechanisms of which are unclear. ${ }^{12}$ Glycogen accumulation in lysosomes is followed by lysosomal leakage or rupture with accumulation of glycogen in cytosol as well as lysosomes. ${ }^{11}$ Lysosomal leakage or rupture also releases hydrolytic enzymes believed to have a role in muscle destruction ${ }^{11}$ with degradation of myofibrils adjacent to glycogen accumulation in the cytosol. ${ }^{12}$ Skeletal muscle contraction may cause lysosomes to rupture in muscle more than in other cell types, contributing to the greater relative involvement of muscle. ${ }^{11}$ Lipofuscin-mediated 
apoptosis has been hypothesized to contribute to muscle wasting. ${ }^{12}$ With disease progression, endstage fibers are left containing empty fluid-filled space with remnants of myofibrillar and sarcoplasmic material. ${ }^{13}$ Large vacuoles are evident in muscle fibers on MRI. ${ }^{14}$ Protein catabolism and abnormal protein metabolism may occur, due to or exacerbated by poor nutrition, with more rapid clearance of branched chain amino acids that normally have a role in muscle protein synthesis. ${ }^{15-18}$ Muscle function may be impaired by loss of contractile mass, decreased contractile function due to clusters of non-contractile material (glycogen) impeding force transmission by interrupting or displacing myofibrils, ${ }^{12,19,20}$ decreased oxidative capacity resulting in decreased adenosine- $5^{\prime}$-triphosphate (ATP) availability for contraction, and impaired innervation. ${ }^{21}$

\section{Clinical presentation and distribution of muscle weakness}

Weakness in Pompe disease is greater in proximal muscles than distal, greater in lower extremities (LE's) than upper extremities (UE's), and generally symmetrical but imbalanced across joints. ${ }^{1}$ Muscles may feel firm or hypertrophic despite profound weakness, ${ }^{1,2}$ especially calf musculature, and sometimes quadriceps, deltoids, and paraspinal muscles.

In the infantile form, progressive, profound, and generalized weakness occurs in neck, trunk, extremities, and facial and oral musculature, with macroglossia (Table 1).,.$^{1,2}$ MRI shows diffuse hypertrophy of muscle groups with large vacuoles but without evidence of fatty infiltration. ${ }^{14}$ Cardiac involvement is severe with early hypertrophic cardiomyopathy progressing later to dilated cardiomyopathy. ${ }^{1,2}$ Respiratory involvement occurs early due to weakness in diaphragm, intercostals, and accessory muscles of respiration, ${ }^{2}$ with ineffective cough due to weakness in abdominals. Paradoxical breathing with sternal and intercostal retraction during inspiration may occur due to weakness in abdominal and intercostal muscles. ${ }^{22}$ (See Pompe Disease Diagnosis and Management Guideline in this issue for respiratory details).

In the late-onset form, progressive proximal myopathy occurs with greater variation in distribution, extent, and rate of progression of weakness, even within families. ${ }^{1,3,4,23,24}$ Patterns of muscle involvement are listed in Table 2. ${ }^{1,3,4,23,25-27}$ With progression, weakness can become profound. ${ }^{7} \mathrm{CT}$ and MRI of muscles in adults with Pompe disease show atrophy, fatty infiltration, and degeneration. ${ }^{25-27}$ Cardiac involvement is not typical in late-onset disease but respiratory involvement may be relatively more severe than skeletal muscle involvement and can be the presenting feature, with apparent selective involvement of the diaphragm. ${ }^{1,4}$

\section{Pathokinesiology of motor function and disease progression}

Pathokinesiology in Pompe disease, as in all motor unit diseases, is characterized by a self-perpetuating cycle in which progressive imbalanced muscle weakness, compensatory movement patterns and postural habits, and the influence of gravity interact in the progression of disability. 28,29 Weakness in Pompe disease evolves from the primary progressive myopathy described above, ${ }^{1,2}$ with possible contribution from anterior horn cell in-

\section{Table 1}

Clinical motor and musculoskeletal presentation in infantile-onset Pompe disease ${ }^{1,2}$

Weakness: "floppy baby" appearance

Profound, progressive, symmetrical weakness

Proximal muscles weaker than distal muscles

Lower extremities weaker than upper extremities

Neck and trunk weakness profound

Facial and oralmotor weakness with myopathic facies

Respiratory involvement includes weakness in: diaphragm, intercostals, accessory muscles of respiration, abdominals

Hypertrophic cardiomyopathy progresses to dilated cardiomyopathy

Positional tendencies:

Hip flexion, abduction, external rotation

Knee flexion

Plantarvarus at feet and ankles

Spinal kyphosis at thoracic and lumbar levels in supported sitting

Head propped back into extension or falling forward in flexion in supported sitting

Forearm pronation, wrist flexion, finger flexion

Sternal and intercostal retraction from paradoxical breathing

Potential secondary musculoskeletal impairments:

Muscle tightness (hypoextensibility)/contracture:

Iliotibial bands (initially seen as lateral thigh groove)

Hip abductors and external rotators

Hip flexors

Hamstrings

Plantarflexors

Plantar fascia

Elbow flexors or extensors (position dependent)

Forearm pronators

Wrist/finger flexors-hypoextensibility over several joints sometimes accompanied by contracture or hypermobility at individual joints

Joint contractures:

Hip flexion, knee flexion, ankle plantarflexion

Elbow flexion or extension (position dependent), wrist and finger flexion

Deformity:

Rib cage flattened in anterior-posterior dimension with:

Sternal retraction (pectus excavatum)

Lower rib flaring

Spinal deformity: kyphosis, scoliosis, lordosis

Pelvic asymmetry with lateral pelvic obliquity, horizontal pelvic rotation, and posterior or anterior pelvic tilt

Hip subluxation/dislocation

Plagiocephaly, brachycephaly, or scaphocephaly

Osteopenia/osteoporosis/fracture 
Table 2

Clinical motor and musculoskeletal presentation in late-onset Pompe disease $\mathrm{e}^{1,3,4,23,25-27}$

Progressive proximal myopathy

Distribution of weakness variable, but:

Proximal muscles generally weaker than distal

Lower extremities generally weaker than upper

Weakness common in:

Pelvic girdle musculature (hip flexors, extensors, abductors, adductors)

Scapular stabilizers

Shoulder girdle musculature (including deltoids)

Spinal extensors (paraspinals)

Neck flexors more than extensors

Abdominals

Hamstrings

Quadriceps

Diaphragm

Weakness may progress to include: biceps brachii, triceps, tongue, and other musculature

Selective early involvement of hip adductors (especially adductor magnus), parapinals, psoas, semi-membranosus, ventrolateral trunk muscles, rectus abdominus, gluteals, vastus medialis

Later involvement of the long head of the biceps femoris, semi-tendinosus, and anterior thigh muscles

Respiratory involvement may be more severe than skeletal muscle involvement, with early involvement of diaphragm

Relative sparing of tensor fascia lata, sartorius, short head of the biceps, gracilis, vastus lateralis, rectus femoris

Risk of secondary musculoskeletal impairment depends on patterns of weakness and compensation and is usually less than in infantile-onset, but scoliosis and lordosis may occur

volvement. Patterns of weakness have been described in Pompe disease ${ }^{1-4,23,24,30}$ and pathokinesiology has been studied in other disorders in which proximal weakness is greater than distal, such as Duchenne muscular dystrophy (DMD), ${ }^{28,31}$ a primary progressive myopathy, and spinal muscular atrophy (SMA), ${ }^{32}$ an anterior horn cell disease. Positioning, postural tendencies, and compensatory patterns of movement in these motor unit diseases are initially determined by the interaction between weakness and gravity, further compromised later by secondary musculoskeletal impairments. ${ }^{28}$ Compensations are effective in maximizing function but, used persistently, can lead to contracture and deformity that contribute to increasing weakness and disability. ${ }^{28}$ Compensations can also limit the use of existing strength by placing muscles at a mechanical disadvantage, compromise length-tension relationships, and limit opportunities to increase or maintain strength, leading to additional disuse atrophy. ${ }^{33}$ Proportional increases in weakness may occur with growth due to biomechanical disadvantage as height, weight, or mass increase without the ability of the muscles to cope with the increased workload, as in DMD and SMA..$^{32}$ Respiratory involvement, and cardiac involvement, if present, can further compromise function and endurance as in other disorders, ${ }^{1,2,34}$ as can undernutrition due to feeding problems from oral-motor weakness. ${ }^{15}$ Detailed descriptions of pathokinesiology in infantile and late-onset disease are included in the electronic version of this article.

\section{Secondary musculoskeletal impairments}

Secondary musculoskeletal impairments occur in accordance with principles of biomechanics and developmental biomechanics ${ }^{35}$ in which the application of small forces over time has powerful effects, especially during periods of rapid growth, with muscle length strongly influenced by the number of hours per day that muscles are in shortened or lengthened positions. ${ }^{36-38}$

\section{Muscle extensibility impairments, contracture, and deformity}

Muscle hypoextensibility, muscle hyperextensibility, contracture, and deformity can develop from the chronic alterations in posture and positioning that result from weakness ${ }^{39}$ and can further compromising muscle function, effective movement, positioning, and comfort. Contracture and deformity occur in accordance with severity of weakness, age of onset and duration of weakness, extent of imbalanced muscle activity, and intervention used or not used to minimize contracture and deformity. ${ }^{28,37}$ Muscles that cross two or more joints are at increased risk of early contracture. ${ }^{28}$ With severe early weakness, the unopposed force of gravity has the most profound effect on positioning and the development of contracture and deformity, as in SMA. ${ }^{40}$ With moderate to mild weakness, muscle imbalance across joints has an increased role in the development of contracture and deformity, as in DMD. ${ }^{28,37}$ Immobilization of muscle from chronic positioning in any diagnosis can lead to changes in fiber length and extensibility, sarcomere number, sarcomere length, length-tension curves, collagen concentration and orientation, ratio of tissue to muscle fiber, stiffness, and changes in ratio of tendon length to muscle belly, ${ }^{36,38}$ with muscle plasticity responsive to both deforming and corrective forces.

Positional tendencies in individuals with infantile-onset Pompe disease lead to early contracture of iliotibial (IT) bands, followed by hip and knee flexion contractures, and contracture of plantarflexors and plantar fascia. Classic areas at risk for abnormal muscle extensibility, contracture, and deformity in the infantile-onset form are listed in Table $1 .{ }^{1,2}$ Risk of contracture and deformity is also present in late-onset Pompe disease, including scoliosis and lordosis, ${ }^{1}$ but presence and severity may vary due to greater variation in weakness. Additional detail is covered in the electronic version of this article.

\section{Osteopenia/osteoporosis}

Osteopenia and osteoporosis have recently been recognized as complications of Pompe disease and osteopenia has been identified in infants as young as four months of age. ${ }^{41}$ Femoral and vertebral fractures have been identified in patients with infantile-onset disease ${ }^{41}$ and vertebral fracture has been reported in late-onset disease ${ }^{42}$ (see Pompe Disease Diagnosis 
and Management Guideline in this issue for details regarding osteopenia and osteoporosis).

\section{Gross motor function}

Infants with infantile-onset disease historically did not achieve motor milestones of independent sitting, creeping, standing, or walking, $, 1,2,43,44$ a fact that is changing with ERT, with some children on ERT now walking independently. ${ }^{2,5,6}$ In a study of physical disability in children spanning infantile and late-onset forms and ranging from age six months to 22 years, $2 / 3$ were non-ambulatory, $3 / 4$ used a ventilator, and age did not correlate with level of disability. ${ }^{45}$

Individuals with late-onset disease characteristically experience gradual difficulty with motor function, noted initially in walking, running, and activities of elevation against gravity such as climbing stairs, rising from the floor, getting up out of a chair, and lifting the arms overhead. ${ }^{24}$ The use of mobility devices such as canes, walkers, manual wheelchairs, and powered mobility are usually added gradually, often used part-time at first, for long distance community mobility or on uneven terrain. ${ }^{24}$ Progression of weakness and increased risk of falls may lead to ambulation only possible or practical indoors on level surfaces for short distances in safe environments such as home; with a manual or motorized wheelchair or scooter used in all other environments. ${ }^{24}$ Mean age at which use of a wheelchair is initiated has been reported as 46 years $^{24}$ and mean age at which ambulation is lost as 50 years. ${ }^{3}$

\section{PHYSICAL THERAPY MANAGEMENT}

Physical therapy (PT) management of Pompe disease, as in all motor unit diseases, should provide comprehensive, anticipatory, and preventative management based on an understanding of the pathophysiology and pathokinesiology of disease presentation and evolution, and on individual assessment. ${ }^{28,33,46}$ The key to management lies in understanding the interaction between the presence, progression, and potential remediation of weakness; the biomechanics of efficient movement; the risks for development of contracture and deformity and strategies for prevention and remediation; the risks and possible remediation of osteoporosis; and function. Muscle weakness presents and progresses in generally predictable patterns with predictable compensations used to cope with weakness. ${ }^{1,2,28}$ Secondary muscle tightness, contracture, and deformity occur in predictable patterns without intervention. ${ }^{28}$ Intervention is focused on breaking or slowing this predictable, self-perpetuating cycle of events whenever possible ${ }^{29}$ so that strength and endurance are maximally achieved and maintained, contracture and deformity are minimized, and compensations can be used to maximize function without leading to increased disability. PT intervention is designed to optimize and preserve motor and physiological function as much as possible within the limits of the disease; to minimize the clinical impact of the disease process; to prevent or minimize secondary complications; to promote and maintain the maximum level of function, functional independence, and participation; to optimize quality of life; and to maximize the benefits of enzyme replacement or other treatments as they become available. PT should address all domains of the World Health Organization (WHO) International Classification of Functioning, Disability, and Health (ICF), ${ }^{47}$ as well as the interaction between these domains; and evaluation and intervention should reflect current standards of care and management. ${ }^{48}$ Selected PT assessment tools are listed in Appendix A of the Pompe Disease Diagnosis and Management Guideline in this issue. PT may be provided in a variety of settings, depending on the needs and preferences of the individual and family (see Pompe Disease Diagnosis and Management Guideline in this issue for details regarding service delivery and funding sources).

\section{Management of skeletal muscle function (strengthening and enhancing motor function)}

Guidelines for muscle strengthening are not established for individuals with Pompe disease. Studies of the effect of strengthening in individuals with Pompe disease have been few, with small numbers, limited to late-onset form, and recommend aerobic and sub-maximal exercise, reporting that sub-maximal exercise may stimulate the degradation of some of the glycogen that accumulates in the cytosol ${ }^{15}$ and that aerobic exercise combined with optimal nutrition may help clear glycogen from muscle with accompanying gains in strength and function. ${ }^{49,50}$ Concern exists, however, about excessively strenuous strengthening, consistent with precautions in disorders characterized by muscle degeneration in which strenuous exercise contributes to weakness by increasing muscle degeneration. ${ }^{34}$ Awareness of general precautions with exercise in the presence of myopathy and cardio-respiratory impairment is important as well as the potential, theoretical concern in Pompe disease that excessive muscle contraction could increase leakage of glycogen from lysosomes or cause lysosomal rupture. ${ }^{11}$ Studies of the effects of strengthening and exercise including strengthening and exercise with ERT are needed. If structural and physiological integrity in muscle is re-established with ERT due to a decrease in abnormal glycogen accumulation, more normal capacity for exercise may emerge. There is early evidence in animals that enzyme replacement may clear glycogen from cardiac and type I muscle fibers more efficiently and more fully than from type II fibers which may impact strengthening emphasis and results. ${ }^{51}$ Until more definitive studies are completed, caution and moderation in exercise are recommended.

Recommendations regarding strengthening of fragile muscles include precautions and guidelines from other degenerative muscle diseases ${ }^{34}$ with an emphasis on the use of sub-maximal and aerobic exercise, avoidance of excessive resistive and eccentric exercise, use of functional activities for exercise, and appropriate monitoring of cardiopulmonary and respiratory response to activity, to exercise, and even to different positions (especially supine). Monitoring with pulse oximetry is recommended during initial examination and treatment, with changes in status and activity, and as needed based on medical stability. Exercise programs should consider fragility due to possible loss of contractile mass and contractile protein content and the possibility of abnormal force transmission within the muscle cells due to accumulation of non-contractile glycogen. ${ }^{12}$ Consideration of physiological fragil- 
ity should include cardio-respiratory impairment and potentially decreased oxidative capacity resulting in decreased ATP availability for muscle contraction. ${ }^{21}$

Intervention for enhancing muscle function should include strategies to optimize biomechanical advantage for movement, minimize contracture against which weak muscle must work, allow practice and strengthening within limits of physiological stability, avoid overwork weakness, avoid disuse atrophy, use energy conservation techniques, and avoid excessive fatigue (Table 3). Although excessively strenuous exercise should be avoided, gentle sub-maximal exercise is believed to be important to allow practice of new motor skills and patterns of functional movement, especially in children; to allow strengthening within physiological limits; to avoid additional disuse atrophy, especially in muscles that might not be used spontaneously because of biomechanical disadvantage and relative weakness compared to other muscles; and to avoid secondary decrease in cardiopulmonary function and deconditioning from inactivity.

\section{Infantile-onset Pompe disease}

Weakness in infants can be profound. Medical stability and ranges of cardiopulmonary safety should be established prior to initiation of therapeutic activity and cardiopulmonary response to activity and to position changes should be monitored. Self-initiated rests should be respected and rests should be established as needed to insure medical stability, avoid overwork weakness, and conserve energy. Positioning and support to maximize biomechanical advantage, decrease the impact of

Table 3

Physical therapy management-movement and strengthening

Establish medical stability and ranges of cardiopulmonary stability

Optimize biomechanical advantage for movement

Provide positioning and support to increase biomechanical advantage

Optimize influence of gravity

Use positioning that optimizes length-tension relationships in muscle

Minimize contracture against which muscles must work

Control degrees of freedom to provide permissive conditions for optimal movement and for the emergence of new movement

Allow practice and gentle strengthening within limits of physiological stability, following precautions and guidelines from other degenerative muscle disease

Use sub-maximal, aerobic exercise

Use active assistance where needed

Avoid overwork weakness and excessive fatigue

Avoid excessive resistance during exercise

Avoid eccentric exercise

Establish rests as appropriate and respect self-initiated rests

Use appropriate cardiopulmonary monitoring during exercise

Avoid disuse atrophy

Use energy conservation techniques gravity, and minimize the development of contracture against which weak muscles must work can allow easier, less strenuous use of weak muscles, increasing the possibility of appropriate practice of developmental skills and avoidance of additional disuse atrophy. Strategies of support during functional exercise should include well-known motor control principles including control of degrees of freedom to allow permissive conditions for the emergence and practice of movement that might not be possible without support. The buoyancy of water in a tub/pool ${ }^{52}$ can provide opportunities for movement while guarding against overexertion, as long as aquatic precautions are followed, especially regarding medical stability and the effects on respiration of the hydrostatic pressure of water on the trunk. Prolonged exposure to excessively heated pools should be avoided in myopathies in order to avoid fatigue. Gentle facilitation of movement with active, graded assistance and with assisted practice of movement in all medically safe positions may allow additional practice of developmental skills and strengthening of muscle within physiological limits and may maximize the benefits of ERT as it becomes available. Specific therapeutic activities making use of the above principles can be used to maximize head and trunk control, facilitate weight shift and transition between positions, facilitate sitting skills and floor mobility such as rolling and creeping on hands and knees, and facilitate standing and walking in children with the capability for these skills. ${ }^{5,6,10}$ Details (Figs. 1,2) regarding therapeutic intervention for these tasks are presented in the online version of this article. Coordination with the rest of the team should occur regarding bone density and integrity, levels of osteoporosis, hip joint status, and potential precautions regarding weight bearing.

\section{Late-onset Pompe disease}

For older individuals, and those with more residual strength, gentle sub-maximal and aerobic functional exercise, such as swimming and cycling without excessive resistance, with active assist as needed, and functional activities, performed with respect for the limitations of cardiopulmonary and muscular endurance are generally considered beneficial, ${ }^{15,34,49,50,52}$ and may maximize the benefits of ERT as it becomes available. Excessive resistive and eccentric exercise is considered contraindicated in most neuromuscular diseases characterized by muscle degeneration, although debate exists over details of appropriate guidelines for many disorders. ${ }^{34}$ Sub-maximal, graded, regularly scheduled exercise is considered beneficial in optimizing strength and avoiding additional disuse atrophy. ${ }^{34}$ Excessive stair climbing is generally considered an activity to avoid in disorders characterized by muscle degeneration because of excessive concentric demands when ascending stairs and excessive eccentric demands when descending stairs. Energy conservation techniques assist in avoiding excessive exertion which may lead to overwork weakness.

\section{Management of respiratory muscle function and pulmonary status}

Respiratory/pulmonary management should follow current established guidelines for neuromuscular disorders, ${ }^{53}$ and 

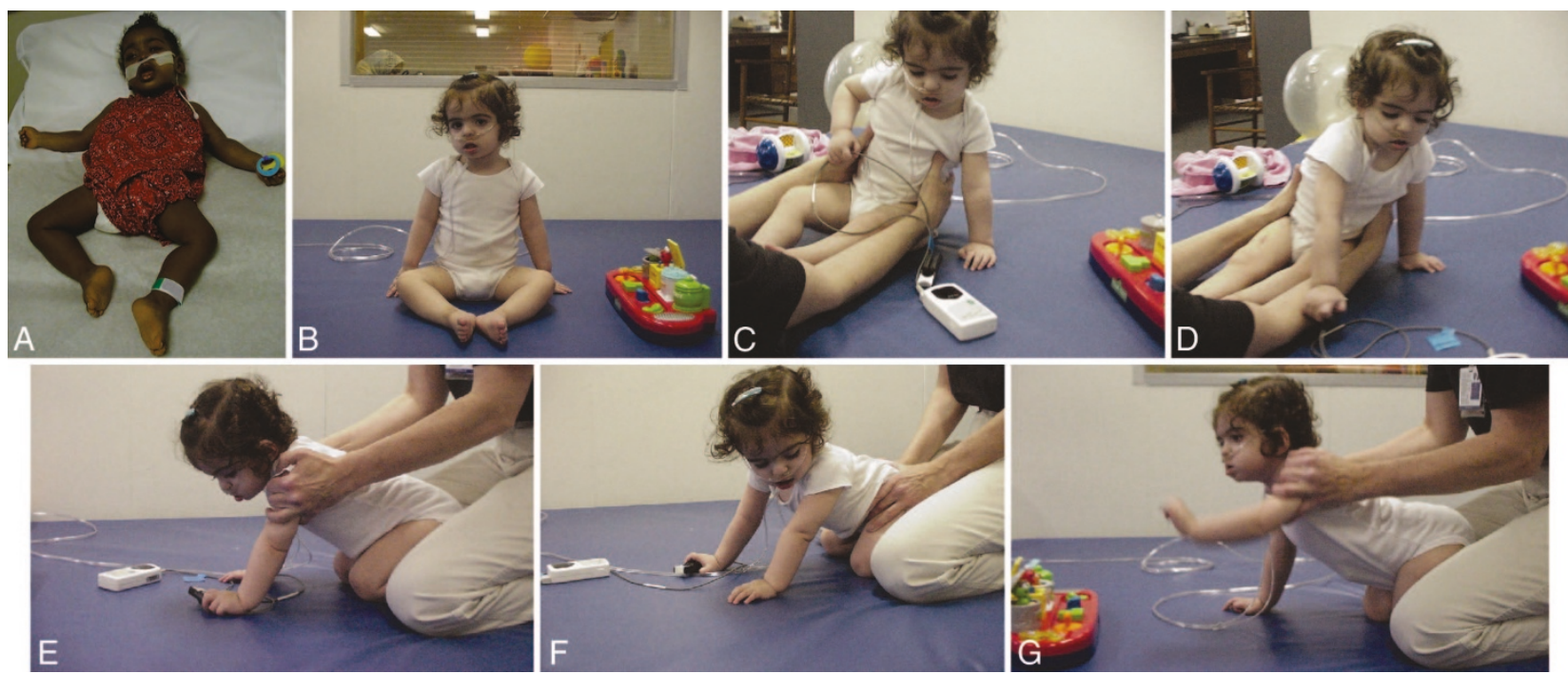

Fig. 1. Classic positioning and facilitation of strengthening and increased function in sitting and quadruped. A) Classic positioning and weakness/hypotonia in infantile-onset Pompe disease. B-G) 14 month old child with infantile-onset Pompe disease on ERT. B) Sitting posture with straight back, but lower extremities in typical flexion, abduction, external rotation (provides stability but prevents weight shift and transition out of sitting). May lead to scooting in sitting for mobility, rather than creeping on hands and knees, if creeping is not facilitated. C) Facilitation of weight shift, protective extension, and weight bearing through upper extremity in sitting, with lower extremity positioning controlled to allow transition to another position. D) Stabilization of lower extremities and facilitation of weight shift in sitting for more active beginning trunk rotation, reaching across midline and outside of the base of support, with weight bearing through the other upper extremity. E) Facilitation of quadruped with support at shoulders and hips. F) Facilitation of quadruped with support only at hips. G) Facilitation of forward weight shift and reaching in quadruped.

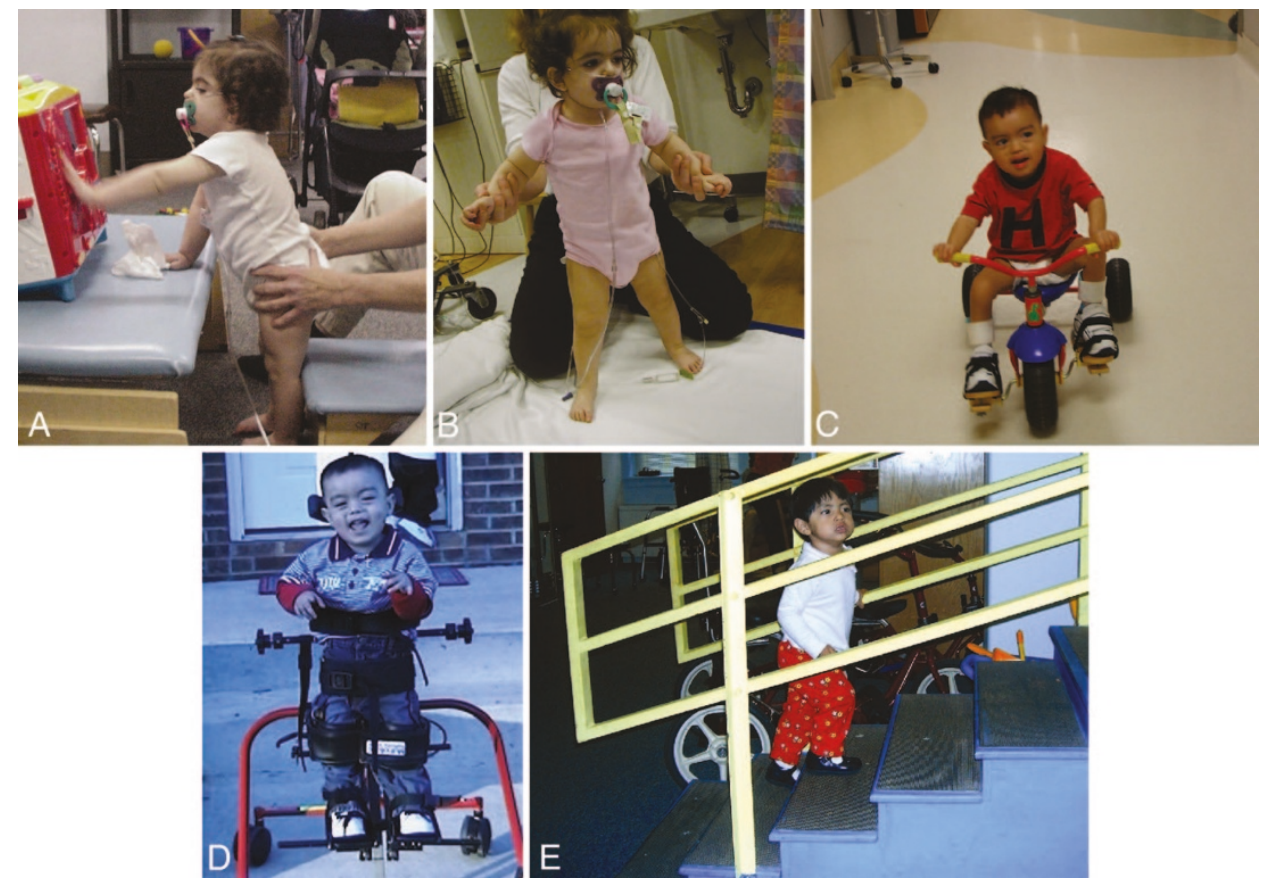

Fig. 2. Facilitation of standing and function with children with infantile-onset Pompe disease on enzyme replacement therapy: A) Facilitation of sit-to-stand and supported standing at a support with 14-month-old child. B) Facilitation of standing with hand support with 14-month-old child. C) Twenty-one-month-old child using adapted tricycle with shoe holders. D) Twenty-one-month-old child using stander for experience in standing, to provide weight bearing in good alignment, and to prevent/minimize contracture into hip flexion, knee flexion, ankle plantarflexion-elongates gastrocnemius muscles across knee and ankle joints simultaneously. E) Independent stair climbing. 
specific guidelines for Pompe disease as outlined in the Pompe Disease Diagnosis and Management Guideline in this issue. Respiratory and pulmonary care is supported by PT management of respiratory/pulmonary function, including recognizing the effects of positioning on respiratory function, maximizing respiratory function and efficiency, use of airway clearance and pulmonary hygiene techniques (potentially including chest PT, active cycle breathing, autogenic breathing, positive expiratory pressure and flutter, intrapulmonary percussive ventilation, high frequency chest wall oscillation (HFCWO) with the use of vests, manually and mechanically assisted coughing with a mechanical in-exsufflator, and appropriate exercise, depending on age, level of involvement, and capabilities of the individual), 54 maintenance of rib cage mobility, and inspiratory muscle training as appropriate and as established in other neuromuscular disorders. Monitoring of oxygenation levels in different positions, and during activity and exercise with pulse oximetry is recommended.

\section{Prevention of secondary musculoskeletal impairments}

Secondary musculoskeletal impairments, including contracture and deformity, should be prevented by counteracting deforming forces in accordance with principles of developmental biomechanics ${ }^{35}$ using gentle forces over time with daily stretching, correction of positioning, splinting and orthotic intervention, provision of adequate support in all positions, support in standing as appropriate, use of adaptive equipment and assistive technology, and education of patients and families (Table 4). ${ }^{55,56}$ Increased incidence of osteopenia and osteoporosis, and increased risk of fracture ${ }^{41}$ must be considered in application of forces. Interventions with the potential to contribute to bone strength and integrity, including weight bearing in PT and standing devices, shown to be associated with increased bone density in individuals with neuromuscular diagnoses, ${ }^{57}$ should be considered, but even normal forces have the capacity to lead to fragility fractures ${ }^{58}$; in osteoporotic bone, and care must be taken in force application. The natural evolution, and effective principles for treatment, of contracture and deformity in neuromuscular disorders are well established ${ }^{28,55}$ and should be followed in intervention for individuals with Pompe disease.

If adequate strength for more normal and complete antigravity movement is available early through ERT, and if motor milestones are achieved at relatively typical ages with a normal amount of movement throughout the day, the forces that lead to contracture and deformity may not occur and prevention of secondary musculoskeletal impairment may occur spontaneously through normal movement, as in typically developing children. However, monitoring and protection of the musculoskeletal system during ERT is critical so that secondary impairments such as contracture and deformity do not occur and limit the benefits of enzyme replacement.

Stretching should be initiated preventatively, done daily when there is a risk of contracture due to weakness and chronic positioning, and is best augmented by follow-up positioning including positional supports, splints and orthotic devices, and adaptive equipment. ${ }^{56,59}$ Stretching should include areas iden-
Table 4

Physical therapy management-prevention of contracture and deformity

Optimize alignment and positioning

Minimize deforming force of gravity

Stretching:

Passive stretching should be done daily and is best augmented by:

Active assistance if possible

Joint mobilization as needed

Modalities as appropriate (gentle heat, warm bath)

Stretch the structures that are at risk, and those identified by individual assessment:

Iliotibial bands

Hip and knee flexors

Hamstrings

Plantarflexors

Plantar fascia

Elbow flexors

Forearm pronators

Wrist/finger flexors

Anything else at risk by exam: individual joints, rib cage, spine

Follow-up with active movement as possible and prolonged positioning

Orthotic intervention (orthoses, casting)

Lower extremity binders (thigh binders)

Knee immobilizers/knee splints

Spine jackets

Wrist/hand/finger flexor splints

Standers or wheelchairs with standing capability

Adaptive seating and mobility devices (specialized strollers and manual and motorized wheelchairs with custom seating and motorized positional changes)

The number of hours per day in a given position determines the development/ prevention of contracture and deformity. Standing may be important to minimize contracture and osteopenia/osteoporosis.

tified by individual assessment and areas of classic risk including hip flexors, IT bands, tensor fascia lata, hamstrings, long and short plantarflexors, posterior tibialis, plantar fascia, forearm pronators, and long wrist and finger flexors; with isolated stretching into hip and knee extension and ankle dorsiflexion. A stretching program is easier to establish as part of the daily routine if it is begun before muscle tightness/contracture is established and before stretching is painful. Stretching should be done gently and physiologically, with an awareness of the risks of fragility fractures ${ }^{58}$ in the presence of ostopenia. Positional supports to augment stretching are detailed in the electronic version of this article and orthotic intervention is outlined below.

Measures to prevent and reduce contracture and deformity require diligence over time and are important to minimize the resistance and biomechanical disadvantage against which weak 
muscles must work and to allow motor function and progression. Consideration must be given to the number of hours per day that a muscle is in a shortened position, as this will determine the risk for the development of contracture and deformity as well as determining successful prevention. ${ }^{36-38}$

\section{Adaptive equipment and orthotic intervention}

Adaptive equipment and orthotic intervention can be used to support function, provide positioning to control contracture and deformity, and allow changes in position and pressure relief for maintenance of skin integrity.

Orthotic intervention (Fig. 3) may include ankle foot orthoses (AFO's) for prevention of plantarflexion contractures, ${ }^{56}$ thigh binders for prevention of IT band contractures, and wrist/hand/finger splints to prevent shortening of long wrist/ finger flexors over multiple joints and flexion contracture at the wrist or in individual finger joints. Knee extension splints may be used to prevent knee flexion contractures and maintain hamstring extensibility.

Seating systems in adapted strollers or wheelchairs are used to assist in preventing or minimizing contracture and deformity, especially spinal deformity, and should include solid seat, solid back, hip guides, lateral trunk supports, knee adductors, and head support as needed. Head support should be in place if the seating system is used during transport in a motor vehicle. Adapted car seats and specialized seat belts, harnesses, and vests can be used for safe support in vehicles, and those designed for infants with poor head control should be used for safe transport of fragile infants.
Supported standing may be beneficial in neuromuscular disease for prevention or minimization of osteoporosis ${ }^{58}$ as well as for contracture control. Recommendations regarding the use of standers are detailed in the electronic version of this article.

The use of positioning, splinting, and standing devices should be coordinated with medical specialists in pediatrics, pulmonary medicine, cardiology, and orthopedics in consideration of potential contraindications in terms of cardiac or pulmonary status, osteoporosis, the risk of fracture, hip subluxation or dislocation, and prohibitive hip and knee flexion contractures.

\section{Function}

At every age, and every stage, appropriate function and maximal levels of independence should be supported as allowed by levels of medical stability, including participation in all aspects of life in which the individual is interested. Functional developmental activities and participation as well as maximal independence in activities of daily living should be supported. Practice, adaptation for function, and family education should be included. Appropriate adaptive equipment and assistive technology should be used to assist in activities of daily living, to maximize function and functional independence, to provide safety in transfers and transport for access. Technology may be the key to function and freedom in many situations, including motorized mobility (with power positioning controls such as tilt, recline, elevating leg rests, seat elevation, seat to floor mobility, and standing) with ventilator trays if needed; power lifts (including portable patient lifts, ceiling lifts, stand-pivot lifts, stair climbers, van lifts); computers (including

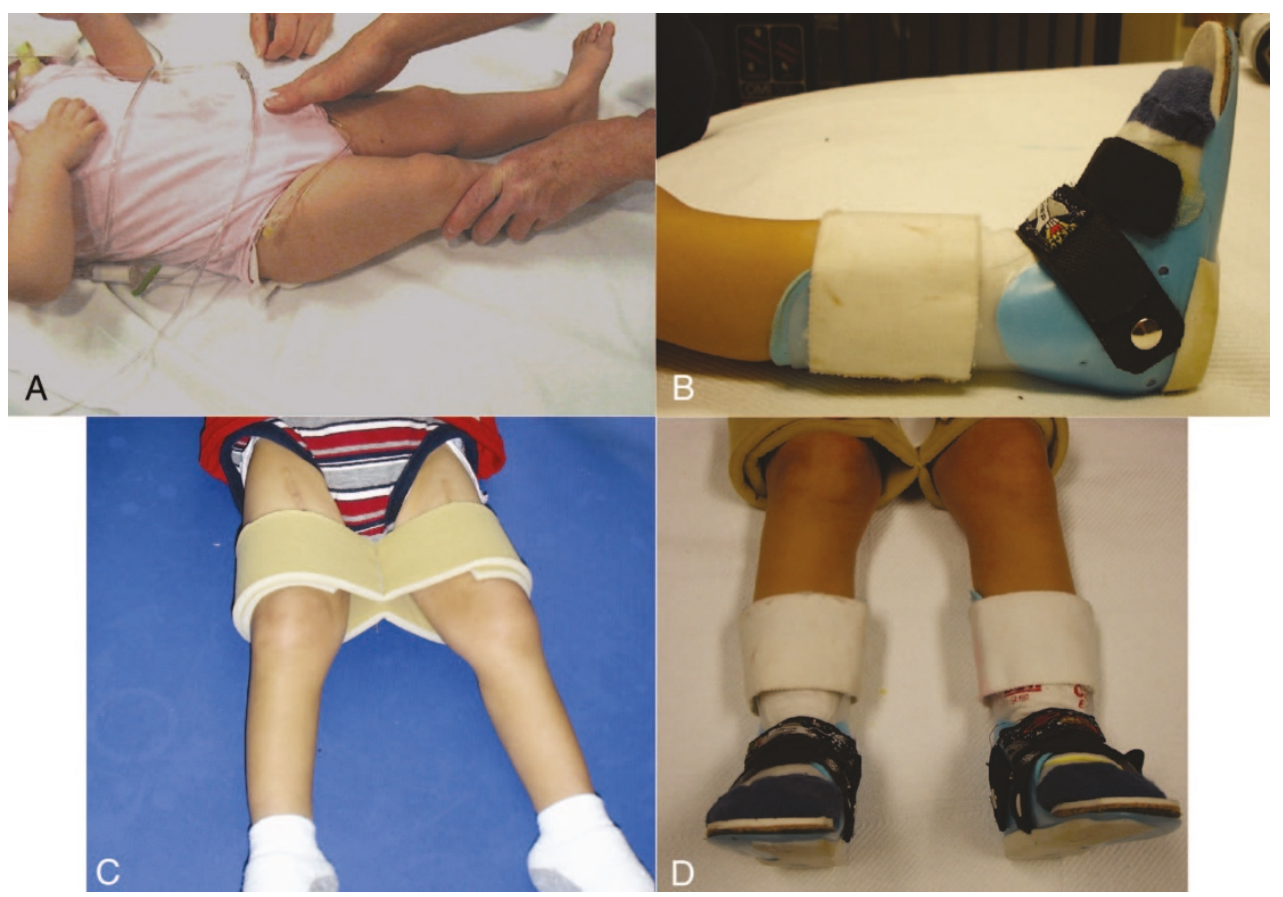

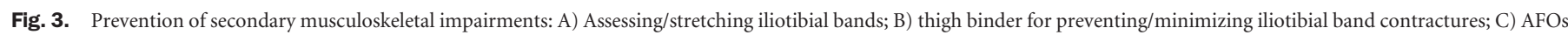

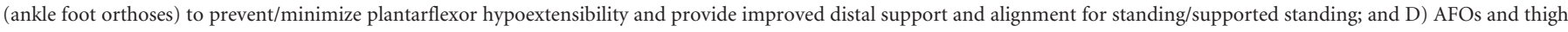
binder used simultaneously to optimize overall lower extremity alignment. 
voice activated systems, adapted keyboards, microswitches, handheld computer devices); internet access; environmental control units; ramps and portable ramps; bathing and bathroom equipment that fosters ease and independence such as specialized bath and shower chairs, hand-held showers, roll-in showers; poweroperated adjustable beds with hand-held remote and alternating pressure pad with pump for pressure relief and maintenance of skin integrity; and all aspects of emerging technology. Motorized mobility should be considered early enough to allow functionally independent mobility at developmentally appropriate ages, reported as early as 20 months of age in SMA. ${ }^{60}$ Recommendation and training in the use of assistive technology at home, school, and work are important. Driving may be appropriate.

\section{CONCLUSION}

The clinical manifestations of Pompe disease are beginning to change with the advent of ERT with greater opportunity for potential improvement in motor status, function, and survival than ever before. ${ }^{5-10}$ With the potential of ERT as a treatment for Pompe disease, provision of comprehensive clinical management is important in maximizing clinical and functional benefits of ERT. The purpose of this article is to increase the understanding of the pathology and pathokinesiology of motor function in Pompe disease, and to provide an overview of physical therapy management. Physical therapy is an important component of management and should be included in the multidisciplinary team for care for individuals with Pompe disease.

\section{ACKNOWLEDGMENTS}

The authors would like to thank Gordon Worley, MD and Kathleen Ollendick Smith, PT, for their thoughtful comments and suggestions in review of the manuscript. This article was written on the authors' own initiative. PSK has received research/ grant support from Genzyme Corporation. LEC has received research support from the Leal Foundation. Both authors have received honoraria from Genzyme Corporation. If therapy for Pompe disease proves successful commercially, Duke University and inventors for the cell line used to generate the enzyme (rhGAA) may benefit financially pursuant to the University's Policy on Inventions, Patents and Technology Transfer.

\section{References}

1. Hirschhorn Reuser AJJ. Glycogen storage disease type II: acid alph $\alpha$-glucosidase (acid maltase) deficiency. In: Scriver ABC, Sly W, et al. (eds.). The Metabolic and Molecular Bases of Metabolic Disease. New York: McGraw Hill;2001:3389-3420.

2. Kishnani PS, Howell RR. Pompe disease in infants and children. J Pediatr 2004;.144: S35-S43.

3. Laforet P, Nicolino M, Eymard PB, Pucch JP, et al. Juvenile and adult-onset acid maltase deficiency in France: genotype-phenotype correlation Neurology 2000;55:11221128.

4. Swash M, Schwartz MS, Apps MC. Adult onset acid maltase deficiency. Distribution and progression of clinical and pathological abnormality in a family. J Neurol Sci 1985;68:61-74.

5. Amalfitano A, Bengur AR, Morse RP, et al. Recombinant human acid alph $\alpha$-glucosidase enzyme therapy for infantile glycogen storage disease type II: results of a phase I/II clinical trial. Genet Med 2001;3:132-138.

6. Van den Hout JM, Kamphoven JH, Winkel LP, et al. Long-term intravenous treatment of Pompe disease with recombinant human alph $\alpha$-glucosidase from milk. Pediatrics 2004;113:448-457.
7. Winkel LP, Van den Hout JM, Kamphoven JH, et al. Enzyme replacement therapy in late-onset Pompe's disease: a three-year follow-up. Ann Neurol 2004;55: 495-502.

8. Kishnani PV, Nicolino T, Tsai M, Herman CH, et al. Enzyme replacement therapy with recombinant Human Acid alpha glucosidase (rhGAA) in InfantilePompe Disease (IPD): results from a Phase 2 study. Pediatric Research 2003;53:A259.

9. Klinge L, Straub V, Neudorf U, Schaper J, et al. Safety and efficacy of recombinant acid alph $\alpha$-glucosidase (rhGAA) in patients with classical infantile Pompe disease: results of a phase II clinical trial. Neuromuscul Disord 2005;15:24-31.

10. Klinge L, Straub V, Neudorf U, Voit T. Enzyme replacement therapy in classical infantile pompe disease: results of a ten-month follow-up study. Neuropediatrics 2005;36:6-11.

11. Griffin JL. Infantile acid maltase deficiency. I. Muscle fiber destruction after lysosomal rupture. Virchows Arch B Cell Pathol Incl Mol Pathol 1984;45:23-36.

12. Hesselink RP, Wagenmakers AJ, Drost MR, Van der Vusse GJ. Lysosomal dysfunction in muscle with special reference to glycogen storage disease type II. Biochim Biophys Acta 2003;1637:164-170.

13. Griffin JL. Infantile acid maltase deficiency. II. Muscle fiber hypertrophy and the ultrastructure of end-stage fibers. Virchows Arch B Cell Pathol Incl Mol Pathol 1984; 45:37-50.

14. Chan WP, Liu GC. MR imaging of primary skeletal muscle diseases in children. AJR Am J Roentgenol 2002;179:989-997.

15. Bembi B, Ciana G, Martini C, Benettoni A, et al. Efficacy of multidisciplinary approach in the treatment of two cases of nonclassical infantile glycogenosis type II. J Inherit Metab Dis 2003;26:675-681.

16. Umpleby AM, Wiles CM, Trend PS, Scobie IN, et al. Protein turnover in acid maltase deficiency before and after treatment with a high protein diet. $J$ Neurol Neurosurg Psychiatry 1987;50:587-592.

17. Umpleby AM, Trend PS, Chubb D, Conaglen JV, et al. The effect of a high protein diet on leucine and alanine turnover in acid maltase deficiency. J Neurol Neurosurg Psychiatry. Aug 1989;52:954-961.

18. Slonim AE, Coleman RA, McElligot MA, Najjar J, et al. Improvement of muscle function in acid maltase deficiency by high-protein therapy. Neurology 1983;33:3438.

19. Hesselink RP, Gorselink M, Schaart G, Wagenmakers AJ, et al. Impaired performance of skeletal muscle in alpha-glucosidase knockout mice. Muscle Nerve 2002; 25:873-883.

20. Drost MR, Hesselink RP, Oomens CW, van der Vusse GJ. Effects of non-contractile inclusions on mechanical performance of skeletal muscle. J Biomech 2005;38:10351043.

21. Lewis SF, Haller RG. Skeletal muscle disorders and associated factors that limit exercise performance. Exerc Sport Sci Rev 1989;17:67-113.

22. Perez A, Mulot R, Vardon G, Barois A, et al. Thoracoabdominal pattern of breathing in neuromuscular disorders. Chest 1996;110:454-461.

23. Felice KJ, Alessi AG, Grunnet ML. Clinical variability in adult-onset acid maltase deficiency: report of affected sibs and review of the literature. Medicine 1995;74:131135.

24. Hagemans ML, Winkel LP, Van Doorn PA, Hop WJ, et al. Clinical manifestation and natural course of late-onset Pompe's disease in 54 Dutch patients. Brain 2005; 128:671-677.

25. Pichiecchio A, Uggetti C, Ravaglia S, Egitto MG, et al. Muscle MRI in adult-onset acid maltase deficiency. Neuromuscul Disord 2004;14:51-55.

26. de Jager AE, van der Vliet TM, van der Ree TC, Oosterink BJ, et al. Muscle computed tomography in adult-onset acid maltase deficiency. Muscle Nerve 1998;21:398-400.

27. Cinnamon J, Slonim AE, Black KS, Gorey MT et al. Evaluation of the lumbar spine in patients with glycogen storage disease: CT demonstration of patterns of paraspinal muscle atrophy. AJNR Am J Neuroradiol 1991;12:1099-1103.

28. Fowler WM. Rehabilitation management of muscular dystrophy and related disorders: II. Comprehensive care. Arch Phys Med Rehabil 1982;63:322-328.

29. Roy L, Gibson DA. Pseudohypertrophic muscular dystrophy and its surgical management: review of 30 patients. Can J Surg 1970;13:13-21.

30. Ausems MG, Wokke JH, Reuser AJ, van Diggelen OP. Juvenile and adult-onset acid maltase deficiency in France: genotype-phenotype correlation. Neurology 2001;57: 1938.

31. Sutherland DH, Olshen R, Cooper L et al. The pathomechanics of gait in Duchenne muscular dystrophy. Dev Med Child Neurol 1981;23:3-22.

32. Iannaccone ST, Russman BS, Browne RH, Buncher CR et al. Prospective analysis of strength in spinal muscular atrophy. DCN/Spinal Muscular Atrophy Group. J Child Neurol 2000;15:97-101

33. Vignos PJ Jr. Physical models of rehabilitation in neuromuscular disease. Muscle Nerve 1983;6:323-338.

34. Fowler WM. Role of physical activity and exercise training in neuromuscular diseases. Am J Phys Med Rehabil 2002;81:S187-S195. 
35. LeVeau BF, Bernhardt DB. Developmental biomechanics. Effect of forces on the growth, development, and maintenance of the human body. Phys Ther 1984;64: 1874-1882.

36. Tardieu C, Lespargot A, Tabary C, Bret MD. For how long must the soleus muscle be stretched each day to prevent contracture? Dev Med Child Neurol 1988;30:3-10.

37. Archibald KC, Vignos Jr. PJ. A study of contractures in muscular dystrophy. Arch Phys Med Rehabil 1959;40:150-157.

38. Baker JH, Matsumoto DE. Adaptation of skeletal muscle to immobilization in a shortened position. Muscle Nerve 1988;11:231-244.

39. Kendall FPaM EK. Muscles-Testing and Function. Baltimore: Williams \& Wilkins; 1983.

40. Garcia-Cabezas MA, Garcia-Alix A, Martin Y, Gutierrez M, et al. Neonatal spinal muscular atrophy with multiple contractures, bone fractures, respiratory insufficiency and 5q13 deletion. Acta Neuropathol (Berl) 2004;107:475-478.

41. Krishnamurthy V, Hanna R, Mackey JM, Dearmy S, et al. Osteopenia in Pompe Disease: A Case Series Presentation. Paper presented at: Society of Inherited Metabolic Disorders; May 2005, 2005; Monterey, CA.

42. Oktenli C. Renal magnesium wasting, hypomagnesemic hypocalcemia, hypocalciuria and osteopenia in a patient with glycogenosis type II. Am J Nephrol 2000; 20:412-417.

43. Kishnani PS, Hwu P, Mandel H, Nicolino M, et al. On behalf of the Infantile-onset Pompe Disease Natural History Study Group-A retrospective, multinational, multicenter study of the natural history of infantile-onset Pompe disease. J Pediatrics (in press).

44. van den Hout HM, Hop W, van Diggelen OP, Smeitink JA, et al. The natural course of infantile Pompe's disease: 20 original cases compared with 133 cases from the literature. Pediatrics 2003;112:332-340.

45. Haley SM, Fragala MA, Skrinar AM. Pompe disease and physical disability. Dev Med Child Neurol 2003;45:618-623.

46. Haley SM. The emerging role of the pediatric physical therapist in evaluation and intervention for individuals with lysosomal storage diseases. Ped Phys Ther 2005; 17: $128-139$.

47. International Classification of Functioning, Disability, and Health (ICF). World Health Organization - 2001. Available at http://www.who.int/classifications/icf/en/. Accessed April 6, 2006.
48. Guide to Physical Therapy Practice. Physical Therapy 2001;81.

49. Slonim AE, Bulone L, Minikes J, Hays AP, et al. Benign course of glycogen storage disease type IIb in two brothers: Nature or nurture? Muscle Nerve 2006;33:571-574.

50. Slonim A, Rosenthal H, O'Connor MR, Goldberg T et al. High protein and exercise therapy (HPET) for childhood acid maltase deficiency (AMD). J Neurolog Sci 1990; 98:465.

51. Raben N, Fukuda T, Gilbert AL, de Jong D, et al. Replacing acid alph $\alpha$-glucosidase in Pompe disease: recombinant and transgenic enzymes are equipotent, but neither completely clears glycogen from type II muscle fibers. Mol Ther 2005;11: 48-56.

52. Figuers C. Aquatic therapy intervention for a child diagnosed with spinal muscular atrophy. Physical Therapy Case Reports 1999;2:109-112.

53. Finder JD, Birnkrant D, Carl J, Farber HJ, et al. Respiratory care of the patient with Duchenne muscular dystrophy: ATS consensus statement. Am J Respir Crit Care Med 2004;170:456-465.

54. Wagener JS, Headley AA. Cystic fibrosis: current trends in respiratory care. Respir Care 2003;48:234-246.

55. McDonald CM. Limb contractures in progressive neuromuscular disease and the role of stretching, orthotics, and surgery. Phys Med Rehabil Clin N Am 1998;9:187211.

56. Hyde SA, FlLytrup I, Glent S, Kroksmark AK, et al. A randomized comparative study of two methods for controlling Tendo Achilles contracture in Duchenne muscular dystrophy. Neuromuscul Disord 2000;10:257-263.

57. Ward K, Alsop C, Caulton J, Rubin C, et al. Low magnitude mechanical loading is osteogenic in children with disabling conditions. J Bone Miner Res 2004;19: 360-369.

58. Biggar WD, Bachrach LK, Henderson RC, Kalkwarf H, et al. Bone health in Duchenne muscular dystrophy: a workshop report from the meeting in Cincinnati, Ohio, July 8, 2004. Neuromuscul Disord 2005; 15:80-85.

59. Stuberg WA. Muscular dystrophy and spinal muscular atrophy. In: Campbell SK, Vander Linden DW, Palisano RJ, ed. Physical Therapy for Children. second ed. Philadelphia: W. B. Saunders Company; 2000:339-368.

60. Jones MA, McEwen IR, Hansen L. Use of power mobility for a young child with spinal muscular atrophy. Phys Ther 2003;83:253-262. 